\title{
Comparative Reaction of Camelina sativa to Sclerotinia sclerotiorum and Leptosphaeria maculans
}

\author{
Maria I. Purnamasari, ${ }^{1,2}$ William Erskine, ${ }^{1,2}$ Janine S. Croser, ${ }^{1,2}$ Ming Pei You, ${ }^{2}$ and Martin J. Barbetti ${ }^{2, \dagger}$ \\ ${ }^{1}$ Centre for Plant Genetics and Breeding, UWA, School of Agriculture and Environment and the UWA Institute of Agriculture, \\ The University of Western Australia, WA, 6009, Australia \\ ${ }^{2}$ School of Agriculture and Environment and the UWA Institute of Agriculture, The University of Western Australia, WA, 6009, \\ Australia
}

\begin{abstract}
Sclerotinia sclerotiorum and Leptosphaeria maculans are two of the most important pathogens of many cruciferous crops. The reaction of 30 genotypes of Camelina sativa (false flax) was determined against both pathogens. C. sativa genotypes were inoculated at seedling and adult stages with two pathotypes of $S$. sclerotiorum, highly virulent MBRS-1 and less virulent WW-1. There were significant differences $(P<0.001)$ among genotypes, between pathotypes, and a significant interaction between genotypes and pathotypes in relation to percent cotyledon disease index (\% CDI) and stem lesion length. Genotypes 370 (\% CDI 20.5, stem lesion length $1.8 \mathrm{~cm}$ ) and 253 (\% CDI 24.8, stem lesion length $1.4 \mathrm{~cm}$ ) not only consistently exhibited cotyledon and stem resistance, in contrast to susceptible genotype 2305 (\% CDI 37.7, stem lesion length $7.2 \mathrm{~cm}$ ),

indicated a complex pattern of inheritance of resistance to $S$. sclerotiorum. Six isolates of $L$. maculans, covering combinations of five different avirulent loci (i.e., five different races), were tested on $C$. sativa cotyledons across two experiments. There was a high level of resistance, with $\% \mathrm{CDI}<17$, and including development of a hypersensitive reaction. This is the first report of variable reaction of $C$. sativa to different races of $L$. maculans and the first demonstrating comparative reactions of $C$. sativa to $S$. sclerotiorum and $L$. maculans. This study not only provides new understanding of these comparative resistances in $C$. sativa, but highlights their potential as new sources of resistance, both for crucifer disease-resistance breeding in general and to enable broader adoption of $C$. sativa as a more sustainable oilseed crop in its own right.
\end{abstract} but their resistance was independent to $S$. sclerotiorum pathotype. A $\mathrm{F}_{5}$-recombinant inbred line population was developed from genotypes $370 \times 2305$ and responses characterized. Low broad-sense heritability
Keywords: Camelina sativa, Sclerotinia sclerotiorum, Leptosphaeria maculans, resistance heritability, disease resistance
Camelina sativa (false flax) has potential to become an important cruciferous oilseed crop due to its high-value products and positive agronomic traits (Campbell et al. 2013). C. sativa oil has a unique fatty acid profile, particularly rich in $n-3$ (omega-3), and has numerous potential markets in food, nutraceuticals, cosmetics, stock feeds, and industrial products (e.g., wax esters) (Iven et al. 2016; Lawrence et al. 2016; Nguyen et al. 2013). However, the greatest potential for C. sativa oil lies in second-generation biofuel, it being a proven lowcost, nonfood biodiesel feedstock in the northern United States (Agusdinata et al. 2011). In addition to its oil value, $C$. sativa has agronomic features that make it an ideal crop per se for sustainable agriculture. Such qualities include its favorable response to low-input farming practices, pod shatter resistance, and drought tolerance (Campbell et al. 2013; Gugel and Falk 2006; Waraich et al. 2013). These traits, along with its short growth cycle, enable $C$. sativa to be sown as an alternative to canola (Brassica napus), in rotation with cereals in semiarid cropping systems and as demonstrated in the Great Plains region of the United States of America (Obour et al. 2015). An additional key trait of $C$. sativa is its resistance to many common pests and pathogens that cause significant yield losses in B. napus (Pavlista et al. 2011; Séguin-Swartz et al. 2009).

${ }^{\dagger}$ Corresponding author: M. J. Barbetti; martin.barbetti@uwa.edu.au

Funding: This work was supported with funding from the Indonesia Endowment Fund for Education Scholarship and The Centre for Plant Genetics and Breeding, The University of Western Australia. Financial assistance to M. Purnamasari was provided through an Indonesia Endowment Fund for Education Scholarship.

The author(s) declare no conflict of interest.

Accepted for publication 16 May 2019.

(C) 2019 The American Phytopathological Society
Sclerotinia rot (SR, causal agent: Sclerotinia sclerotiorum) and blackleg (also known as phoma stem canker, causal agent: Leptosphaeria maculans) are the two most important diseases of crucifers worldwide, including canola and mustard (Brassica juncea) (Barbetti et al. 2015; Delourme et al. 2012; Li et al. 2008; Sivasithamparam et al. 2005; Uloth et al. 2013). Both pathogens can infect at any stage of plant/crop development (Khangura and Barbetti 2001; Li et al. 2008; Uloth et al. 2013, 2014, 2015). In Australia, yield loss from either disease has on occasions exceeded 50\% (GRDC 2013; Sivasithamparam et al. 2005), and SR alone causes losses of AU\$ 23 million in Western Australia (DAFWA 2015). In comparison with canola and mustard, relatively few studies have addressed either disease in $C$. sativa, despite $C$. sativa genotypes known to show variation for resistance to SR (e.g., Eynck and Séguin-Swartz 2009). Using a high genetic diversity $C$. sativa germplasm collection (Ghamkhar et al. 2010), we previously showed variable response to SR across $30 C$. sativa genotypes at the seedling stage, observing a range of response from mildly susceptible to resistant (Purnamasari et al. 2015). However, there can be inconsistency in the expression of resistance against SR depending upon the type of inoculation test adopted (Uloth et al. 2013, 2014) and/or pathotype (Barbetti et al. 2014; Ge et al. 2012; Neik et al. 2017; Willbur et al. 2017). Previously, Ge et al. (2012) identified 8 distinct $S$. sclerotiorum pathotypes from 53 isolates obtained from the agricultural regions of Western Australia, isolates taken from infested stems of canola and lupin that showed severe Sclerotinia stem rot disease. Accordingly, it is important when defining SR resistance in $C$. sativa to use more than one type of inoculation test and to compare resistance expression utilizing contrasting pathotypes. Therefore, there remained a clear need to further explore resistance against $S$. sclerotiorum within this collection of $C$. sativa and to determine the mechanism of resistance to SR. Toward this aim, subsequent to earlier research of Purnamasari et al. (2015), we first developed a 141 genotype recombinant inbred line (RIL) population from resistant $\times$ susceptible parents identified in that earlier study.

C. sativa has also been reported to exhibit a very high level of resistance to L. maculans (Salisbury 1987; Séguin-Swartz et al. 2009). 
For example, Li et al. (2005) found $C$. sativa R4175-01W2 developed no symptoms to 80 isolates of L. maculans. Similarly, Gregorich et al. (2009) found no disease symptoms in four varieties of $C$. sativa inoculated with two races of $L$. maculans. Field trials have confirmed $C$. sativa to be very highly resistant or immune to blackleg disease (Séguin-Swartz et al. 2009). However, notably, these historical studies have only involved relatively few genotypes and/or $L$. maculans races. Hence, there was a clear need to also evaluate a diverse $C$. sativa collection for responses to inoculation with a wider range of L. maculans races.

Toward meeting these needs, studies were undertaken to: 1) determine the reaction of 30 diverse genotypes of $C$. sativa against two different pathotypes of $S$. sclerotiorum at cotyledon and adult stages and different races of L. maculans at cotyledon stages; and 2) characterize (at the cotyledon stage) $141 \mathrm{~F}_{5-6}$ RILs derived from crossing a resistant with a susceptible genotype of $C$. sativa, for their responses to $S$. sclerotiorum. We discuss new understanding of comparative resistances to these diseases in C. sativa and highlight the value of potential new sources of resistance to both diseases for breeding across a range of oilseed and horticultural crucifers.

\section{Materials and Methods}

Fungal isolates. The isolates of $S$. sclerotiorum and L. maculans used in this experiment are listed in Table 1. Criteria for selecting the isolates were based on their differences in pathogenic potential on $C$. sativa. For $S$. sclerotiorum, two different pathotypes were chosen: 1) isolate MBRS-1, a very aggressive isolate belonging to the prevailing pathotype (pathotype 76) occurring in Western Australia (Ge et al. 2012), which has been used extensively for screening of crucifers (e.g., Uloth et al. 2013) and for C. sativa (Purnamasari et al. 2015); 2) isolate WW-1 (pathotype unknown), a less aggressive isolate that causes distinctly different relative resistance/susceptibility rankings across $B$. napus genotypes as compared with MBRS-1 (Garg et al. 2010). For L. maculans, isolates chosen were: UWA192 (preliminary experiment), as it is a highly virulent isolate on $B$. napus genotypes containing single dominant gene-based resistance derived from Brassica rapa subsp. sylvestris (Li et al. 2004a). Isolates UWAM3, UWAP11, WAC4028, WAC4094, and WAC7803 (second experiment) were chosen based on their avirulent (Avr) loci for which the isolate is avirulent and represented races Av1-4-5-7-8, Av1-5-6-7, Av3-5-6, Av1-3-5-6-8, and Av6-9 (Balesdent et al. 2005). Furthermore, isolate UWAP11 is also known to be highly virulent on B. napus genotypes containing polygenic resistance ( $\mathrm{Li}$ et al. 2003).

Plant materials. Thirty genotypes of $C$. sativa obtained from the N.I. Vavilov Research Institute of Plant Industry, Russia, originating from five countries (former Czechoslovakia, France, Russia, Sweden, and Ukraine), were evaluated (Table 2). These genotypes are well characterized in relation to agronomic performance, fatty acid analysis, molecular, ecogeographic analysis, and their cotyledon resistance against S. sclerotiorum MBRS-1 (Campbell et al. 2013; Ghamkhar et al. 2010; Purnamasari et al. 2015). B. napus 'Mystic' and 06P712 were used as check comparisons in S. sclerotiorum studies, and 'Mystic' was used as a check comparison in L. maculans studies. The response of B. napus 'Mystic' and 06P712 to S. sclerotiorum has previously been defined with isolates MBRS-1 and/or WW-1 (Garg et al. 2010; Ge et al. 2012; Li et al. 2006; Uloth et al. 2013, 2014, 2015; You et al. 2016). Furthermore, in the current study, a 141-line $\mathrm{F}_{5}$-RIL population was established by single seed descent from a cross between resistant $C$. sativa 370 and susceptible

Table 2. Mean stem lesion length (cm) of 30 Camelina sativa and Brassica napus genotypes inoculated with different isolates of Sclerotinia sclerotiorum (MBRS-1 and WW-1). A unique relative ranking score for each genotype is provided within brackets, where 1 represents the most resistant genotype and the largest relative genotype ranking score represents the most susceptible genotype. $^{\mathrm{a}}$

\begin{tabular}{|c|c|c|c|c|c|c|}
\hline \multirow{3}{*}{$\frac{\text { Genotype }}{253}$} & \multirow{3}{*}{$\frac{\text { Origin }}{\text { Russia }}$} & \multirow{3}{*}{$\frac{\text { Species }}{\text { C. sativa }}$} & \multicolumn{4}{|c|}{$\begin{array}{l}\text { Mean lesion length }(\mathrm{cm}) \\
\text { and relative } \\
\text { ranking score }\end{array}$} \\
\hline & & & \multicolumn{2}{|c|}{ MBRS-1 } & \multicolumn{2}{|c|}{ WW-1 } \\
\hline & & & 1.6 & (1) & 1.2 & (14) \\
\hline 344 & Russia & C. sativa & 1.7 & (2) & 1.3 & (19) \\
\hline 4059 & Russia & C. sativa & 1.7 & (3) & 1.5 & (25) \\
\hline 2504 & Russia & C. sativa & 1.8 & (4) & 1.9 & (26) \\
\hline 4177 & $\begin{array}{l}\text { Former } \\
\text { Czechoslovakia }\end{array}$ & C. sativa & 1.9 & (5) & 1.5 & (23) \\
\hline Mystic & Australia & B. napus & 2.3 & (6) & 1.1 & (11) \\
\hline 06Р712 & China & B. napus & 2.4 & (7) & 1.1 & (12) \\
\hline 235 & Russia & C. sativa & 2.5 & (8) & 0.8 & (5) \\
\hline 339 & Russia & C. sativa & 2.5 & (9) & 1.3 & (17) \\
\hline 1993 & Russia & C. sativa & 2.6 & (10) & 0.4 & (2) \\
\hline 370 & Russia & C. sativa & 2.7 & (11) & 0.9 & (6) \\
\hline 3364 & Ukraine & C. sativa & 2.8 & (12) & 1.0 & (9) \\
\hline 2292 & Russia & C. sativa & 2.8 & (13) & 0.4 & (1) \\
\hline 4112 & Russia & C. sativa & 2.8 & (14) & 3.4 & (32) \\
\hline 349 & Russia & C. sativa & 2.9 & (15) & 1.4 & (21) \\
\hline 4182 & Russia & C. sativa & 3.0 & (16) & 1.0 & (10) \\
\hline 4139 & Russia & C. sativa & 3.2 & (17) & 1.5 & (24) \\
\hline 1811 & France & C. sativa & 3.5 & (18) & 1.1 & (13) \\
\hline 430 & Russia & C. sativa & 3.9 & (19) & 1.0 & (8) \\
\hline 4183 & Russia & C. sativa & 4.1 & (20) & 0.5 & (3) \\
\hline 4138 & Russia & C. sativa & 4.3 & (21) & 1.3 & (20) \\
\hline 4077 & Russia & C. sativa & 4.4 & (22) & 2.2 & (29) \\
\hline 1330 & Russia & C. sativa & 4.9 & (23) & 0.9 & (7) \\
\hline 3347 & Ukraine & C. sativa & 4.9 & (24) & 1.2 & (16) \\
\hline 4130 & Ukraine & C. sativa & 5.0 & (25) & 0.7 & (4) \\
\hline 4068 & Russia & C. sativa & 5.1 & (26) & 1.3 & (18) \\
\hline 2495 & Russia & C. sativa & 6.0 & (27) & 1.2 & (15) \\
\hline 4111 & Russia & C. sativa & 6.1 & (28) & 2.0 & (27) \\
\hline 403 & Russia & C. sativa & 6.4 & (29) & 1.4 & (22) \\
\hline 4164 & Sweden & C. sativa & 7.4 & (30) & 2.0 & (28) \\
\hline 4074 & Russia & C. sativa & 8.6 & (31) & 2.7 & (30) \\
\hline 2305 & Russia & C. sativa & 11.7 & (32) & 2.7 & (31) \\
\hline Mean & & & 4.0 & & 1.4 & \\
\hline
\end{tabular}

${ }^{a}$ Significance of genotypes for MBRS- $1 P<0.001 ; 1$.s.d. $(P<0.05)=3.7$. Significance of genotypes for WW-1 $P<0.001$; 1.s.d. $(P<0.05)=0.8$. Significance of isolates $P<0.001 ; 1$. s.d. $(P<0.05)=0.5$. Significance of genotypes $\times$ isolates $P<0.001 ; 1$. s.d. $(P<0.05)=2.7$.

Table 1. Sclerotinia sclerotiorum and Leptosphaeria maculans isolates used in this study

\begin{tabular}{lllll}
\hline Isolate code & \multicolumn{1}{c}{ Species } & Date & \multicolumn{1}{c}{ Origin } & Source (isolate located) \\
\hline MBRS-1 & S. sclerotiorum & 2004 & Mount Barker, Western Australia & Infected stem tissue of B. napus \\
WW-1 & S. sclerotiorum & 2004 & Walkaway, Western Australia & Infected stem tissue of B. napus \\
UWA192 & L. maculans & 2002 & Mount Barker, Western Australia & Infected stem tissue of B. napus cv. Hyola 60 \\
UWAM3 & L. maculans & 2001 & Mount Barker, Western Australia & Infected stem tissue of B. juncea cv. Roy 394 \\
UWAP11 & L. maculans & 2001 & Wongan Hills, Western Australia & Infected stem tissue of B. napus cv. Pinnacle \\
WAC4028 & L. maculans & 1984 & Mount Barker, Western Australia & Infected stem tissue of B. napus cv. Wesreo \\
WAC4094 & L. maculans & 1984 & Western Australia & Infected stem tissue of B. napus cv. Wesway \\
WAC7803 & L. maculans & 1973 & Western Australia & Infected leaf tissue of Raphanus raphanistrum \\
\hline
\end{tabular}


C. sativa 2305. The two parents for this population were chosen based on their response to $S$. sclerotiorum MBRS-1 using cotyledon inoculation (Purnamasari et al. 2015). All plants were grown in 1-liter pots in a pasteurized soil mixture composed of finely crushed pine bark/coco peat/sand at 2.5:1.0:1.5 (wt/wt/wt). Plants were watered daily and fertilized weekly using Thrive all-purpose soluble fertilizer at the recommended rate. All experiments were conducted within a growth room set to $18 / 14^{\circ} \mathrm{C}$ (day/night) and with a 16 -h light/8-h dark cycle and light intensity of $320 \mu \mathrm{mol}$ quanta $\mathrm{m}^{-2} \mathrm{~s}^{-1}$.

Screening tests for $S$. sclerotiorum. Screening test was carried out using two different methods: 1) cotyledon inoculation for isolate WW-1; and 2) stem inoculation for isolates MBRS1 and WW-1. Inoculum preparation and cotyledon assay were performed as described by Garg et al. (2008). Briefly, two plants per pot were grown in 30-cell trays (85-ml Kwikpot Trays, each cell $55 \mathrm{~mm}$ in diameter) until cotyledons were fully expanded, equivalent to growth stage 1.00 on the Sylvester-Bradley and Makepeace (1984) scale. Seven agar plug discs were cut from actively growing margins of 3 -day-old colonies of $S$. sclerotiorum growing on potato dextrose agar at $20^{\circ} \mathrm{C}$ and were used to inoculate $150 \mathrm{ml}$ of sterilized potato dextrose broth containing peptone (potato dextrose broth $24 \mathrm{~g}$, peptone $10 \mathrm{~g}, \mathrm{H}_{2} \mathrm{O} 1$ liter). Cultures were placed on a rotary shaker at $150 \mathrm{rpm}$ at $20^{\circ} \mathrm{C}$. After 3 days, colonies of $S$. sclerotiorum were collected and washed twice with deionized water. The fungal mats were then transferred to $125 \mathrm{ml}$ of the same liquid medium and macerated using a hand-blender for $3 \mathrm{~min}$. The mycelial suspension was filtered through four layers of cheesecloth to remove any large mycelial components, the density was determined using a hemocytometer, and concentration was adjusted to $1 \times 10^{5}$ fragments $\mathrm{ml}^{-1}$.

For cotyledon inoculation, a single 5- $\mu$ l droplet of WW-1 mycelium suspension was deposited on each lobe of each cotyledon of the 30 C. sativa genotypes using a micropipette. The inoculum was shaken often to maintain a homogenous mycelial suspension. Inoculum suspension contained $0.002 \%$ Tween 20 wetting agent to help cotyledons retain the droplets, and Tween was also included with deionized water used to inoculate control plants of all genotypes. Plants were kept in the dark in 35-liter clear plastic storage boxes with a $2.5-\mathrm{cm}$ depth of water at the bottom of the boxes to maintain high humidity conditions after inoculation. Disease development was assessed at $72 \mathrm{~h}$ postinoculation for $S$. sclerotiorum on 0-9 disease severity scale as used previously by Purnamasari et al. (2015), where: $0=$ no visible symptoms, $1=$ necrotic hypersensitive, $2=$ necrotic or water-soaked lesion ( $10 \%$ of total leaf area), $3=$ necrotic or watersoaked lesion $(20 \%), 4=$ necrotic or water-soaked lesion $(20$ to $30 \%), 5=$ necrotic or water-soaked lesion (30 to 40\%), $6=$ necrotic or water-soaked lesion (40 to $50 \%), 7=$ necrotic or water-soaked lesion $(50$ to $60 \%), 8=$ collapsing of cotyledon tissue, and $9=$ collapsing of cotyledon tissue with masses of mycelium. The disease scores were converted into a percent cotyledon disease index (\% CDI) using the method of McKinney (1923), as follows: \% CDI $=\{[(\mathrm{a} \times 0)+(\mathrm{b} \times$ $1)+(\mathrm{c} \times 2)+(\mathrm{d} \times 3)+(\mathrm{e} \times 4)+\ldots(\mathrm{j} \times 9)] \times 100\} /[\mathrm{a}+\mathrm{b}+\mathrm{c}+\mathrm{d}+\ldots \mathrm{j}) \times 9]$, where $a, b, c, d, e \ldots j$ are the numbers of plants with disease scores of $0,1,2,3,4, \ldots 9$, respectively. There were four replications arranged in complete randomized block design, and the entire experiment was fully repeated once.

Stem inoculation of $S$. sclerotiorum was carried out in a controlled environment using the method described by Li et al. (2006), which is a combination of the methods used by Li et al. (2004b) and Buchwaldt et al. (2005). About $5 \mathrm{~mm}$ diameter mycelial disks from an actively growing 48-h culture grown on glucose-rich medium (peptone $10 \mathrm{~g}$, glucose $20 \mathrm{~g}$, agar $18 \mathrm{~g}, \mathrm{KH}_{2} \mathrm{PO}_{4} 0.5 \mathrm{~g}, \mathrm{H}_{2} \mathrm{O} 1$ liter, pH 6.0 before autoclaving) was placed onto the stem above the first node by wrapping with Parafilm tape. The same diameter disk of glucoserich medium without fungal inocula was used for control treated plants. Five plants of each genotype were used for inoculation when $50 \%$ of the plants had at least one open flower. Plants were irrigated by overhead misting immediately postinoculation for $10 \mathrm{~min}$ and the misting repeated on each of the following 3 days to maintain conducive conditions for S. sclerotiorum. Stem lesion lengths were measured with a linear ruler 3 weeks after inoculation, as this particular time provides disease data that are independent from plant maturity ( $\mathrm{Li}$ et al. 2007a). The experiment was arranged as a complete randomized block design and fully repeated once.

Screening tests for $\mathbf{L}$. maculans. Screening test for $L$. maculans was carried out in two experiments. Experiment 1 was an initial experiment using 30 genotypes of $C$. sativa with $L$. maculans UWA192, and the results of this experiment enabled the selection of six genotypes that were rated as resistant (two genotypes), intermediate (two genotypes), and susceptible (two genotypes) based on the rank order of \% CDI (LSD test). These six genotypes were again challenged with five isolates covering five different races of L. maculans to confirm the response of $C$. sativa to these races (Experiment 2). Conidial suspensions for $L$. maculans were prepared as described by Li et al. (2005). Agar strips $(0.5 \times 1 \mathrm{~cm})$ from actively growing cultures containing mature pycnidia were each transferred to $1 \mathrm{ml}$ deionized water and left until a suspension of conidia was evident. V8 agar plates (V8 juice $150 \mathrm{ml}, \mathrm{CaCO}_{3} 1.5 \mathrm{~g}$, agar $15 \mathrm{~g}, \mathrm{H}_{2} \mathrm{O}$ 1 liter) were spread evenly with $100 \mu$ l of the conidial suspension and incubated at $22^{\circ} \mathrm{C}$ under a single cool-white fluorescent light tube and a single black light tube. After 7 days, the culture was flooded with $10 \mathrm{ml}$ of deionized water and gently rubbed with a glass rod. The conidial suspension was filtered with Mira cloth (Calbiochem, La Jolla, U.S.A.), density determined with a hemocytometer, and the concentration adjusted to $1 \times 10^{7}$ spores $\mathrm{ml}^{-1}$.

Fully developed cotyledons (equivalent to Sylvester-Bradley [1984] growth stage 1.00) of C. sativa were inoculated using standard procedures as developed for B. napus (Li et al. 2005). Cotyledons were punctured once with a stainless-steel needle before inoculation at the puncture point by deposition of $5 \mu l$ of the conidial suspension onto each half-cotyledon. Disease severity was scored 14 days postinoculation using a scale modified from Williams (1985): $0=$ no visible symptoms, $1=$ necrotic hypersensitive, $2=$ small lesion expanding in inoculation spot $(<0.5 \mathrm{~mm}), 3=$ small lesion expanding in inoculation spot $(0.5-1 \mathrm{~mm}), 4=$ collapsed spot $(1 \mathrm{~mm}), 5=$ collapsed spot $(2-3 \mathrm{~mm}), 6=$ collapsed $\operatorname{spot}(3-4 \mathrm{~mm})$, 7 = collapsed spot $(4-5 \mathrm{~mm}), 8=$ collapsed $\operatorname{spot}(>5 \mathrm{~mm})$, and $9=$ cotyledon has died. The disease scores were converted into a percent cotyledon disease index (\% CDI) as described above. For each experiment, there were four replications arranged in complete randomized block design, and the entire experiment was repeated once.

Statistical analyses. GenStat software (18th ed.; VSN International) was used for statistical analysis of data. The statistical $t$ test in GenStat was used to compare the disease data from the original and repeat experiments in each study. As there were no differences between the experiments detected (i.e., $P>0.05$ ), data for both experiments in each study were pooled and analyzed as a single dataset. The \% CDI for S. sclerotiorum MBRS-1 (Table 3) was extracted from Purnamasari et al. (2015). The \% CDI for cotyledons and lesion length for stems were analyzed using analysis of variance (ANOVA). Fisher's least significant differences $(P<0.05)$ were used to separate significant differences between means and calculate the genotype rank order. The latter was a unique relative ranking score for each genotype, provided within brackets, where 1 represents the most resistant genotype and the largest relative genotype ranking score represents the most susceptible genotype. This rank order then was used to classify the genotypes into the most resistant genotypes, intermediateresistant, and the most susceptible genotypes. Regression analysis was undertaken using the regression function in Microsoft Excel to determine the relationship between cotyledon assays and stem inoculation. The broad-sense heritability $\left(\mathrm{H}^{2}\right)$ for \% CDI was calculated from the ANOVA table by a method described by Fehr (1991), as follows: $H^{2}=(\mathrm{GV} / \mathrm{PV}) \times 100 \% ; \mathrm{EV}=\mathrm{MS}_{\mathrm{r}} ; \mathrm{GV}=\left(\mathrm{MS}_{\mathrm{g}}-\mathrm{MS}_{\mathrm{r}}\right) / \mathrm{R} ; \mathrm{PV}=$ $\mathrm{EV}+\mathrm{GV}$, where $\mathrm{GV}$ is genotypic variance, $\mathrm{PV}$ is phenotypic variance, $\mathrm{EV}$ is environmental variance, $\mathrm{MS}_{\mathrm{r}}$ is mean square error, $\mathrm{MS}_{\mathrm{g}}$ is mean square genotype, and $\mathrm{R}$ is the number of replicates.

\section{Results}

Response of $C$. sativa genotypes to $S$. sclerotiorum. Stem inoculation. For plants inoculated with $S$. sclerotiorum isolates 
MBRS-1 and WW-1, there were significant effects $(P<0.001)$ of genotype with regard to the severity of stem lesions (expressed as lesion length) at 3 weeks postinoculation (wpi) (Table 2, Fig. 1). For MBRS-1, genotype 253 was the most resistant with mean stem lesion length $1.62 \mathrm{~cm}$, while genotype 2305 was the most susceptible with lesion length $11.73 \mathrm{~cm}$. For WW-1, the most resistant was genotype 2292 with stem lesion length of $0.4 \mathrm{~cm}$, and the most susceptible was genotype 4112 with stem lesion length of $3.4 \mathrm{~cm}$. There were differences in virulence between the two $S$. sclerotiorum isolates $(P$ $<0.001)$ across both $C$. sativa and $B$. napus genotypes. Isolate MBRS-1 was more virulent with a mean stem lesion length of $4 \mathrm{~cm}$ as compared with WW-1 with a mean stem lesion length of $1.4 \mathrm{~cm}$. Moreover, there was a significant interaction between genotype and isolates $(P<0.001)$, indicating that the genotype response is influenced by isolate pathotype. For example, genotype 4412 was a middle-ranking genotype against MBRS-1 but ranked 32nd for WW-1 based on lesion length (LSD test, Tables 2 and 3). However, some especially susceptible genotypes (e.g., 4074 and 2305) were similarly susceptible against either isolate. In addition, B. napus 'Mystic' and 06P712 were among the most resistant genotypes from B. napus or C. sativa, with mean stem lesion length approximately 2.3 for MBRS-1 and 1.1 for WW-1.

On $C$. sativa, symptoms included wilting of leaves as early as 4 days postinoculation (dpi) and a necrotic and bleached lesion by 1 wpi. At 3 wpi, the most resistant response showed as a very small lesion, accompanied with a hypersensitive-type reaction (Fig. 1A and B). In contrast, stems of some other C. sativa genotypes showed intermediate-sized necrotic lesions ( 2 to $\leq 6 \mathrm{~cm}$ length), demonstrating moderate resistance (Fig. 1C-E). The most susceptible and severely affected plants were observed after inoculation with MBRS-1, and showed the lesion extending and girdling the stem $(>6 \mathrm{~cm})$, causing stem collapse and plant death (Fig. 1F and G).

Cotyledon inoculation: Response of 30 C. sativa genotypes against two S. sclerotiorum isolates. Cotyledon lesion data for isolate WW-1 (current study) and by way of comparison, isolate MBRS-1 (extracted from Purnamasari et al. 2015) are presented (Table 3). There were differences between genotypes in relation to \% CDI at 3 dpi following inoculation with pathogenic versus less pathogenic isolates $(P<0.001)$, as was observed with stem inoculation. Based on the \% CDI values, the most resistant genotypes were 370, 1993, and 253 , with mean $\%$ CDI ranging from 20.5 to 24.8 , whereas 2305 was the most susceptible genotype, with mean \% CDI 37.7. There were also differences between the two isolates $(P<0.001)$, with mean \% CDI for MBRS-1 of 51.7 compared with mean \% CDI for WW-1 of 6.3. Furthermore, there was a significant host $x$ isolate pathotype interaction for cotyledon inoculation, similarly as reported above for stem inoculation $(P=0.002)$. In addition, $B$. napus 'Mystic' was found to fall within the susceptible group, with a mean $\%$ CDI of 34.

Response of $\mathrm{C}$. sativa RILs population against $\mathrm{S}$. sclerotiorum $M B R S-1$. The response of the set of $141 \mathrm{~F}_{5-6}$ RIL population to $S$. sclerotiorum was also evaluated along with resistant parent (C. sativa 370 ) and susceptible donor ( $C$. sativa 2305 ). There were significant genotypic differences between the tested RILs as well as between the parents for their resistance responses to cotyledon inoculation $(P<$ $0.001)$. The parents were consistently different in terms of their reaction to $S$. sclerotiorum, with the resistant versus susceptible parent having \% CDI values of 41.3 and 68.1, respectively. The \% CDI of the RIL population lines ranged from 29.5 to 82 , with the majority falling into the range 50-70 (Fig. 2). Of the RILs evaluated, 30 RILs were considered to belong to the resistant category. The broad-sense heritability of S. sclerotiorum resistance was $36 \%$, which indicated that there was a low genetic variance for SR resistance in $C$. sativa.

Comparison of stem and cotyledon inoculation assays. There was a significant quadratic relationship between mean stem lesion length and \% CDI for MBRS-1 ( $r=0.57, P<0.001, n=31$; Fig. 3$)$. The relative rankings of some genotypes were similar for mean stem lesion length and \% CDI, and for this, examples included genotypes 253, 4111, and 2305 (Tables 2 and 3). In contrast, regression analysis showed no significant relationship between mean stem lesion length and \% CDI where the less virulent $S$. sclerotiorum isolate WW-1 had been used $(r=0.22)$.

Response of $\boldsymbol{C}$. sativa genotypes to $\boldsymbol{L}$. maculans. Experiment 1 . The response of 30 genotypes of $C$. sativa screened against $L$ maculans isolate UWA192 in the initial pathogenicity experiment included various symptoms such as a small dark brown/black necrotic area around the point of inoculation on cotyledons (Fig. 4), and the lesions ranged from these necrotic hypersensitive lesions to larger brown necrosis $(\leq 1 \mathrm{~mm})$ around the inoculated area. Despite the relatively small differences in lesion size, these genotypic differences were significant $(P<0.001)$ in relation to \% CDI by $14 \mathrm{dpi}$ (Table 4). B. napus 'Mystic', the positive inoculation comparison, showed the greatest disease reaction with \% CDI of 61.5. Among C. sativa genotypes, 2305 had the greatest \% CDI (13.9 at $14 \mathrm{dpi}$ ), while 4139 was lowest with a \% CDI of 7.6.

Experiment 2. To further investigate the variability in resistance against $L$. maculans, six $C$. sativa genotypes (two each of resistant, intermediate, and susceptible genotypes) were screened using five different L. maculans isolates, representing races Av1-4-5-7-8, Av1-5-6-7, Av3-5-6, Av1-3-5-6-8, and Av6-9. There was a significant effect of fungal isolate, genotype, and an isolate $\times$ genotype interaction (all $P<0.001$ ) in terms of the disease responses across the

Table 3. Percent cotyledon disease index (\% CDI) of 30 Camelina sativa genotypes and Brassica napus 'Mystic' inoculated with Sclerotinia sclerotiorum isolates (MBRS-1 or WW-1). A unique relative ranking score for each genotype is provided within brackets, where 1 represents the most resistant genotype and the largest relative genotype ranking score represents the most

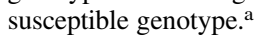

\begin{tabular}{|c|c|c|c|c|}
\hline \multirow{3}{*}{$\frac{\text { Genotype }}{370}$} & \multicolumn{4}{|c|}{$\% \mathrm{CDI}$ and relative ranking score } \\
\hline & \multicolumn{2}{|c|}{ MBRS-1 $^{\mathrm{b}}$} & \multicolumn{2}{|c|}{ WW-1 } \\
\hline & 38.9 & (1) & 2.1 & (4) \\
\hline 1993 & 41.7 & (2) & 5.9 & (14) \\
\hline 4068 & 42 & (3) & 9 & (29) \\
\hline 430 & 45.4 & (4) & 4.5 & (6) \\
\hline 349 & 46.2 & (5) & 5.2 & (10) \\
\hline 253 & 47.9 & (6) & 1.7 & (3) \\
\hline 4139 & 48.3 & (7) & 8.3 & (23) \\
\hline 4059 & 48.6 & (8) & 8.7 & (28) \\
\hline 4112 & 49.3 & (9) & 5.2 & (9) \\
\hline 344 & 50.4 & (10) & 7.6 & (20) \\
\hline 4130 & 51 & (11) & 6.9 & (18) \\
\hline 403 & 51.4 & (12) & 1.4 & (2) \\
\hline 4182 & 51.7 & (13) & 5.9 & (12) \\
\hline 4074 & 51.7 & (14) & 8 & (22) \\
\hline 3364 & 52 & (15) & 4.2 & $(5)$ \\
\hline 235 & 52 & (16) & 1.4 & (1) \\
\hline 4183 & 52.8 & (17) & 6.3 & (15) \\
\hline 4164 & 52.8 & (18) & 8 & (21) \\
\hline 1330 & 52.8 & (19) & 7.6 & (19) \\
\hline 339 & 53.1 & (20) & 4.5 & (7) \\
\hline 3347 & 53.1 & (21) & 8.7 & (27) \\
\hline 2495 & 53.8 & (22) & 6.6 & (16) \\
\hline 4077 & 53.8 & (23) & 6.9 & (17) \\
\hline 1811 & 54.2 & (24) & 5.2 & (11) \\
\hline 2504 & 54.5 & (25) & 10.4 & (30) \\
\hline 4177 & 54.5 & (26) & 10.4 & (31) \\
\hline 4138 & 56.3 & (27) & 4.5 & (8) \\
\hline 4111 & 56.6 & (28) & 8.7 & (25) \\
\hline 2292 & 57.3 & (29) & 8.7 & (26) \\
\hline Mystic & 59.7 & (30) & 8.3 & (24) \\
\hline 2305 & 69.4 & (31) & 5.9 & (13) \\
\hline Mean & 51.7 & & 6.3 & \\
\hline
\end{tabular}

a Significance of genotypes for MBRS-1 $P<0.001 ; 1$. s.d. $(P<0.05)=9.9$ Significance of genotypes for WW-1 $P<0.001$; 1.s.d. $(P<0.05)=4$. Significance of isolates $P<0.001 ; 1$. s.d. $(P<0.05)=1$.4. Significance of genotypes $\times$ isolates $P=0.002$; 1.s.d. $(P<0.05)=7.9$.

${ }^{\mathrm{b}}$ Data extracted from Purnamasari et al. (2015). 
genotypes and isolates tested (Table 5). Overall, all genotypes of $C$. sativa were highly resistant to all isolates, with mean $\% \mathrm{CDI}<17$. In comparison, B. napus 'Mystic' was moderately to highly susceptible to all test isolates, as evidenced by the development of large necrotic lesions, with a mean \% CDI of 66.4. Furthermore, among the L. maculans isolates, UWAP11 caused the most severe disease symptoms, with mean \% CDI of 25.6, while WAC4094 caused least disease symptoms with mean \% CDI of 18 . All L. maculans isolates caused a hypersensitive response (HR) on most $C$. sativa genotypes that was categorized as a disease score of 1 (Fig. 4B). Moreover, nonspreading lesions could also be observed at some infection sites (Fig. 4C and D). In some plants in some genotypes, all isolates except WAC4094 caused severe infection with a score of 4 (Fig. 4E). The responses of some $C$. sativa genotypes were isolate-dependent, such as genotypes 370,3364 , and 4139 . In contrast, genotype 2305 was consistently susceptible in relation to \% CDI against the four isolates used in this study.

\section{Discussion}

Our study provides an evaluation of the relative resistances in a set of 30 genetically diverse genotypes of $C$. sativa to two important diseases in the Brassicaceae, SR and blackleg. We were able to confirm the results of our cotyledonary testing against SR with stem inoculation, confirm resistance of $C$. sativa genotypes against two distinct pathotypes providing new evidence of the high value of

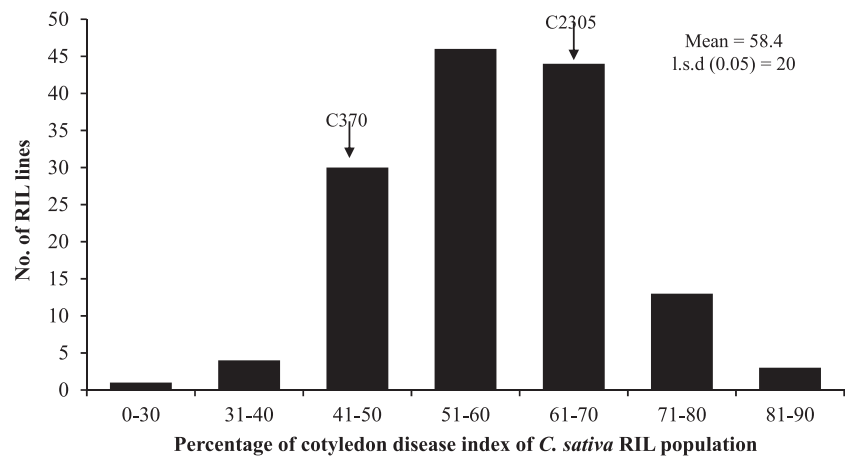

Fig. 2. The proportion for 141 genotypes from the Camelina sativa $370 \times 2305$ recombinant inbred line (RIL) population against Sclerotinia sclerotiorum.
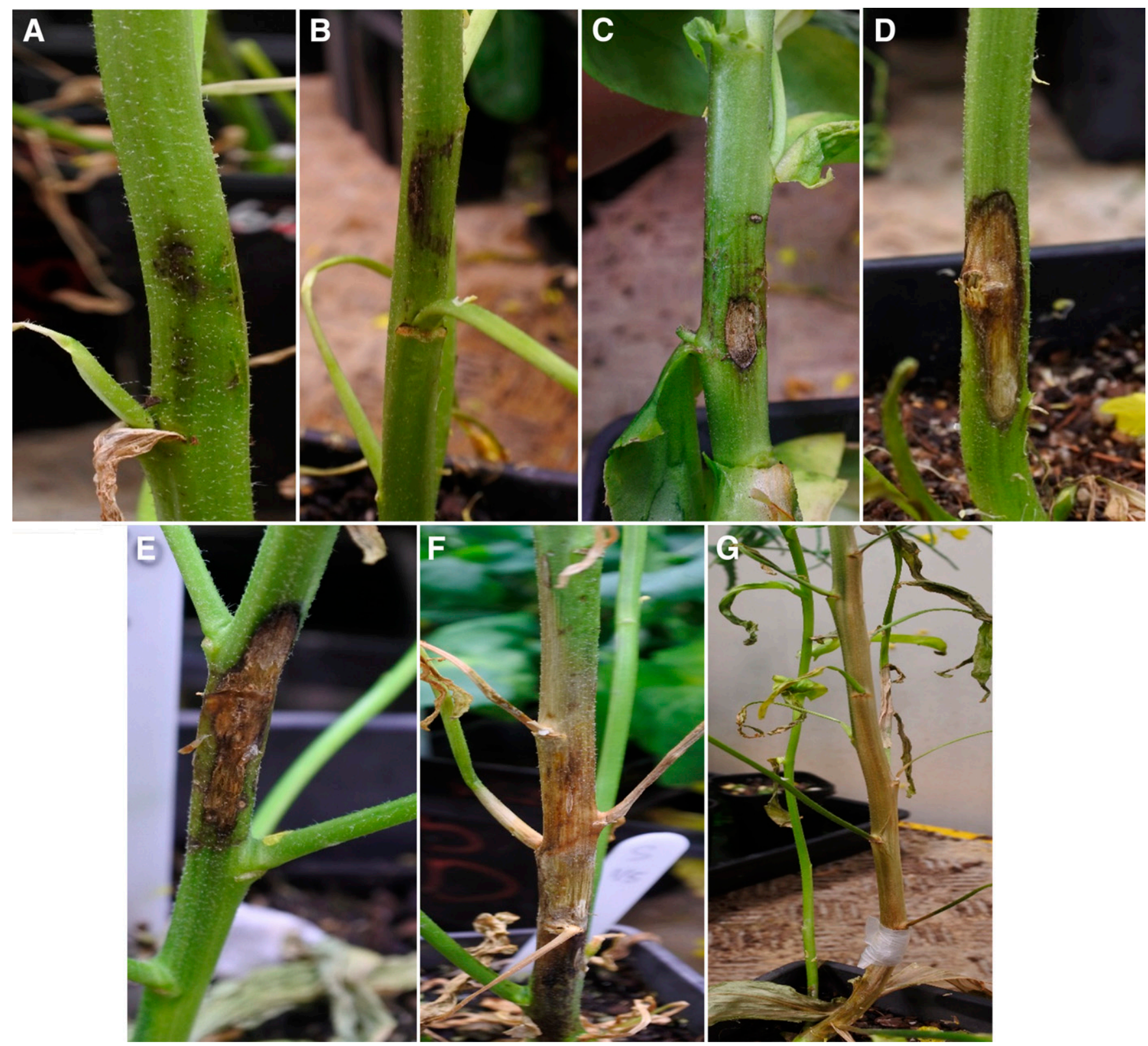

Fig. 1. The range of stem lesion symptoms on Camelina sativa genotypes caused by Sclerotinia sclerotiorum. $\mathbf{A}$ and $\mathbf{B}$ show hypersensitive-type reaction, displaying the high level of resistance. $\mathbf{C}$ to $\mathbf{E}$ show intermediate-sized stem lesions representing moderate/intermediate resistance. $\mathbf{F}$ and $\mathbf{G}$ show a large lesion girdling the stem, indicating extreme susceptibility. 
C. sativa as a resistance source to this pathogen. Additionally, the current study confirmed the outstanding performance of $C$. sativa against $L$. maculans, with genotypes displaying a highly resistant reaction to L. maculans (\% CDI 7.6-18.8). The current study highlighted the six isolates of $L$. maculans tested, representing a diverse range of different Avr allele combinations, readily elicited a $\mathrm{HR}$ reaction. We believe this is the first report of variable $C$. sativa response to $L$. maculans isolates; in contrast to other studies that found no symptoms on $C$. sativa following $L$. maculans inoculation (e.g., Gregorich et al. 2009; Li et al. 2005; Salisbury 1987).

For $S$. sclerotiorum, this study builds upon our previous cotyledonary stage inoculation testing of $C$. sativa (Purnamasari et al. 2015). In the field, stems at the flowering stage are severely damaged by SR, and the stem inoculation method we used provided the first realistic measure for SR resistance on $C$. sativa. Further, previous studies have emphasized the importance of carrying out a range of tests with plants at different stages of maturity (Bradley et al. 2006; Neik et al. 2017; Taylor et al. 2015), as we have done. Our study highlights the importance of using distinct pathotypes of S. sclerotiorum

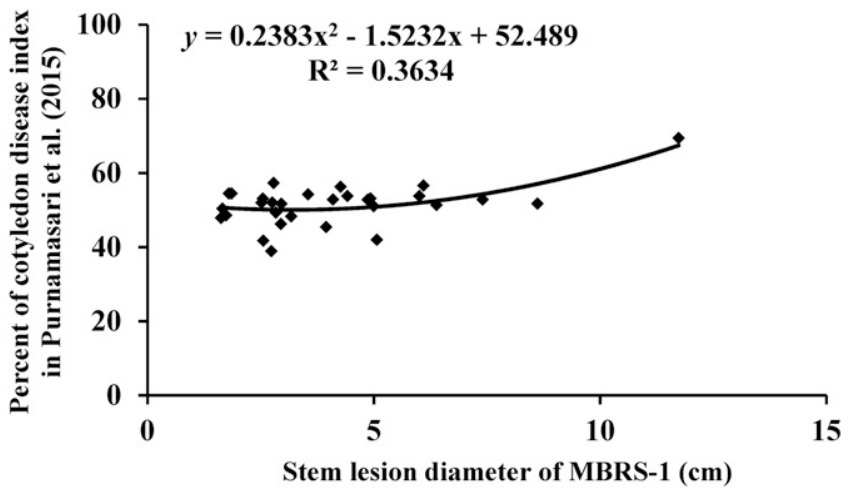

Fig. 3. Correlation of data for stem lesion length 3 weeks after inoculation and $\% \mathrm{CDI} 3$ days after inoculation on 30 genotypes of Camelina sativa when inoculated with Sclerotinia sclerotiorum MBRS-1. Note: \% CDI data has been extracted from Purnamasari et al. (2015).
Table 4. Severity of disease on cotyledons (\% CDI) of 30 Camelina sativa genotypes and Brassica napus 'Mystic' following inoculation with Leptosphaeria maculans UWA192. A unique relative ranking score for each genotype is provided within brackets, where 1 represents the most resistant genotype and the largest relative genotype ranking score represents the most

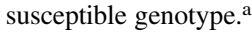

\begin{tabular}{llr}
\hline Genotype & \% CDI and relative ranking score \\
\hline 4139 & 7.6 & $(1)$ \\
430 & 8.3 & $(2)$ \\
1330 & 8.7 & $(3)$ \\
370 & 9 & $(4)$ \\
4183 & 9 & $(5)$ \\
4130 & 9.4 & $(6)$ \\
4177 & 9.4 & $(7)$ \\
4068 & 10.8 & $(8)$ \\
3347 & 11.1 & $(9)$ \\
4077 & 11.1 & $(10)$ \\
2504 & 11.1 & $(11)$ \\
4112 & 11.5 & $(12)$ \\
4164 & 11.5 & $(13)$ \\
3364 & 11.5 & $(14)$ \\
403 & 11.8 & $(15)$ \\
4138 & 11.8 & $(16)$ \\
339 & 11.8 & $(17)$ \\
349 & 12.2 & $(18)$ \\
1993 & 12.2 & $(19)$ \\
253 & 12.2 & $(20)$ \\
1811 & 12.2 & $(21)$ \\
344 & 12.5 & $(22)$ \\
4074 & 12.5 & $(23)$ \\
4059 & 12.9 & $(24)$ \\
4182 & 12.9 & $(25)$ \\
235 & 12.9 & $(26)$ \\
2292 & 13.2 & $(27)$ \\
2495 & 13.2 & $(28)$ \\
4111 & 13.2 & $(29)$ \\
2305 & 13.9 & $(31)$ \\
Mystic & 61.5 & $(30)$ \\
\hline & & 4.5 \\
49 & &
\end{tabular}

${ }^{\text {a }}$ Significance of genotypes for $\%$ CDI: $P<0.001$; 1.s.d. $(P<0.005)=4.04$.

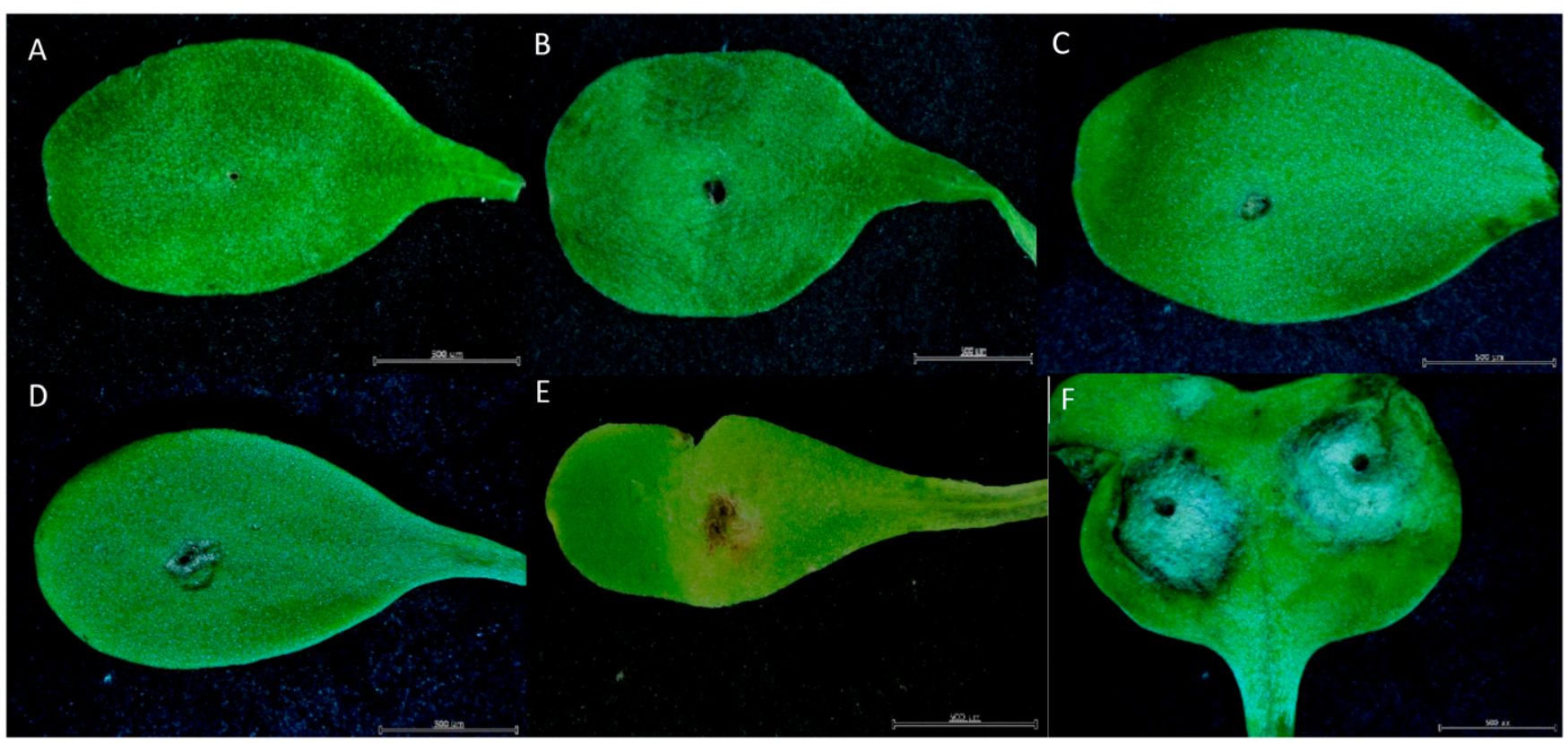

Fig. 4. Responses on cotyledons of Camelina sativa and Brassica napus to inoculation with Leptosphaeria maculans. A, No symptoms formed on C. sativa (disease score $=0$ ). B Necrotic hypersensitive reaction (disease score $=1$ ). $\mathbf{C}$, Very small necrotic tissue surrounding the inoculation point on $\mathbf{C}$. sativa (disease score $=2$ ). $\mathbf{D}$, Small necrotic tissue around the inoculation point on C. sativa (disease score $=3$ ). E, Necrotic tissue with diameter $1 \mathrm{~mm}$ on C. sativa (disease score $=4$ ). F, Disease symptoms on B. napus 'Mystic' (disease score $=7$ ). 
with varying abilities to overcome particular host resistance(s) and/or associated resistance mechanisms of certain genotypes, and in order to identify pathotype-independent host resistance in $C$. sativa.

Overall, we found $C$. sativa genotypes expressed good resistance, with 18 genotypes having stem lesions 1.6 to $3.5 \mathrm{~cm}$, equivalent to the top-ranked resistances for the two B. napus genotypes tested, 'Mystic' and 0P6712. Previously, using the same stem inoculation technique, 'Mystic' and 06P712 were found to be the top-ranked resistant B. napus genotypes tested in Uloth et al. (2015) and in You et al. (2016). Although the relative resistance rankings of $C$. sativa genotypes varied depending upon different inoculation technique and/or isolates, some genotypes (highly resistant or highly susceptible) displayed consistent responses regardless of the inoculation technique or pathotype of S. sclerotiorum. However, other genotypes with moderate level resistance, such as genotypes 2292 and 4068, were less consistent across different screening studies. This inconsistency is to be expected for genotypes with moderate level resistance as environment influences resistance expression (Sun et al. 2005, Uloth et al. 2013). However, You et al. (2016) did identify a few Chinese $B$. oleracea var. capitata genotypes that expressed extremely high-level combined stem and leaf resistance. Genotypes 370 and 253 will be particularly significant for developing new SR-resistant C. sativa genotypes, as they have resistance mechanism(s) that appear to be effectively independent of environmental settings, to $S$. sclerotiorum pathotypes, and/or plant component (i.e., cotyledon or stem).

There was noteworthy correlation between mean stem lesion length and the mean \% CDI across $C$. sativa genotypes for $S$. sclerotiorum MBRS-1, although not for WW-1. This is supported by Garg et al. (2008), who showed cotyledon resistance was well correlated with stem resistance for a set of $B$. napus genotypes. The correlation across different plant components (e.g., cotyledon or stem) makes these highly resistant $C$. sativa genotypes potential sources of resistance to target and exploit in developing new commercial Brassicaceae cultivars with more effective combined seedling and adult plant resistance. This outcome is particularly significant as many other studies have shown cotyledon, leaf, and stem resistances are generally expressed differently across different plant components. For example, You et al. (2016) found no correlation between expressions of stem versus leaf resistance, suggesting independent inheritance. Similarly, Uloth et al. (2013) found no correlation between seedling cotyledon and adult plant stem resistance following artificial inoculation, nor with naturally occurring leaf infection across a similar diverse range of cruciferous genotypes in field studies. The correlation in the current study between cotyledon and stem resistance in C. sativa for highly virulent isolate of $S$. sclerotiorum suggests a common basal resistance mechanism that operates against this pathogen in both plant stages. Eynck et al. (2012) has shown monolignol biosynthesis is linked with $S$. sclerotiorum resistance in $C$. sativa, and a strong induction of monolignol genes in resistant genotypes enhances lignin synthesis at the pathogen inoculation site, thus restricting the development and expansion of the pathogen within the plant. Similarly, Uloth et al. (2016) highlight the importance of lignin production in impeding $S$. sclerotiorum reaching the stem vascular and xylem tissues in highly resistant $B$. carinata, B. juncea, and B. napus. Further studies to confirm the role of monolignol genes in resistance in $C$. sativa to $S$. sclerotiorum would be instructive.

When we challenged the susceptible $\times$ resistant RIL population by inoculation at the cotyledonary stage, the $141 \mathrm{~F}_{5}$ RILs differed for $\%$ CDI following $S$. sclerotiorum inoculation. However, the estimate of broad-sense heritability for SR resistance in this population was $36 \%$; lower than in previous studies, such as the $67 \%$ found in $B$. napus by Zhao et al. (2006) in relation to stem resistance (using petiole inoculation) or the $61 \%$ found by Wu et al. (2013) in relation to leaf resistance (at seedling stage). Nevertheless, the high heritability values in those studies were not reflected when dissecting the genetic variance responsible for a phenotypic variation of SR resistance. These and other studies found all QTLs identified through biparental RIL mapping studies to be minor effect QTLs, explaining $\leq 10 \%$ of the variance for SR resistance (Wei et al. 2014; Wu et al. 2013; Yin et al. 2009; Zhao et al. 2006). Similarly, genome wide association studies (GWAS) for SR resistance identified only a few loci that collectively explain $16.5 \%$ of the phenotypic variance while the observed broad-sense heritability was $61.7 \%$ (Wu et al. 2016). All of the available studies and the low broad sense heritability of resistance to $S$. sclerotiorum in $C$. sativa suggest the resistance mechanism for SR in this species is a very complex genetic trait determined by many genes each of little effect. One strategy to uncover the molecular mechanism for resistance to $S$. sclerotiorum in $C$. sativa may be to identify candidate genes through the histological approach of transcriptomic sequencing followed by a candidate gene approach. For example, by using candidate gene-based association mapping strategy, Rana et al. (2017) found that marker-trait associations could explain $30 \%$ of the phenotypic variation in B. juncea and B. fruticulosa introgression lines to $S$. sclerotiorum.

In the current study, the outstanding resistance of $C$. sativa genotypes to L. maculans (\% CDI 7.6-18.8) and isolate independence of this reaction was confirmed. Previously, $C$. sativa, as a distantly related species of canola, has been reported to exhibit high resistance to L. maculans in field trials (Salisbury 1987; Séguin-Swartz et al. 2009). Furthermore, Li et al. (2005) found that 80 isolates of $L . m a c$ ulans did not cause any symptoms on a single $C$. sativa genotype. Similarly, Gregorich et al. (2009) found that there were no disease symptoms in four varieties of $C$. sativa inoculated with two specific races of $L$. maculans that were virulent to $B$. napus. Our study also highlighted the six isolates of $L$. maculans tested, representative of races with diverse Avr allele combinations, readily elicited a HR. This outcome may be due to the inherent genetic diversity present in our $C$. sativa panel, a germplasm collection known to have higher genetic variability than previously reported from other $C$. sativa germplasm reports (Ghamkhar et al. 2010) or to the relatively limited genotypes used in previous studies. Bohman et al. (2004) found a single genotype of Arabidopsis thaliana that showed evident disease symptoms to L. maculans out of 168 genotypes. Nevertheless, in the current study, as all $C$. sativa genotypes showed a strong resistance reaction and the disease symptoms were greatly restricted compared with the $B$. napus control, it could be concluded that $C$. sativa

Table 5. Response of six Camelina sativa genotypes for five different races of Leptosphaeria maculans. A unique relative ranking score for each genotype is provided within brackets, where 1 represents the most resistant genotype and the largest relative genotype ranking score represents the most susceptible genotype. ${ }^{\mathrm{a}}$

\begin{tabular}{|c|c|c|c|c|c|c|c|c|c|}
\hline \multirow[b]{2}{*}{ Isolate } & \multirow[b]{2}{*}{ Race $^{b}$} & \multicolumn{8}{|c|}{ Host } \\
\hline & & 370 & 430 & 2305 & 3364 & 4111 & 4139 & Mystic & Mean \\
\hline UWAM3 & Av1-4-5-7-8 & $13.2(4)$ & $12.5(3)$ & $16.3(6)$ & $11.5(1)$ & $13.5(5)$ & $12.2(2)$ & 71.9 (7) & 21.6 \\
\hline UWAP11 & Av1-5-6-7 & $16(3)$ & $17(5)$ & $18.4(6)$ & $14.6(2)$ & $16.3(4)$ & $11.1(1)$ & $86.1(7)$ & 25.6 \\
\hline WAC4028 & Av3-5-6 & $11.5(1)$ & $12.5(2)$ & $18.8(6)$ & $15.6(5)$ & $13.5(3)$ & $14.2(4)$ & $47.2(7)$ & 19.1 \\
\hline WAC4094 & Av1-3-5-6-8 & $10.4(1)$ & $10.4(2)$ & $13.2(6)$ & $10.8(4)$ & $10.4(3)$ & $12.2(5)$ & $58.3(7)$ & 18 \\
\hline WAC7803 & Av6-9 & $18.4(6)$ & $13.5(3)$ & $16.3(5)$ & $11.1(2)$ & $16(4)$ & $11.1(1)$ & $73.6(7)$ & 22.9 \\
\hline
\end{tabular}

${ }^{a}$ Significance of genotypes for UWAM3; $P<0.001 ; 1 . s . d .(P<0.005)=8.6$. Significance of genotypes for UWAP11; $P<0.001 ; 1$. s.d. $(P<0.005)=6.3$. Sig nificance of genotypes for WAC4028; $P<0.001 ; 1$. s.d. $(P<0.005)=10$. Significance of genotypes for WAC4094; $P<0.001 ; 1$. s.d. $(P<0.005)=4.8$. Significance of genotypes for WAC7803; $P<0.001 ;$ 1.s.d. $(P<0.005)=7.3$. Significance of $L$. maculans isolates; $P<0.001 ; 1 . s . d$. $(P<0.005)=2.7$. Significance of isolates $\times$ genotypes; $P<0.001 ;$ 1.s.d. $(P<0.005)=7.1$.

${ }^{\mathrm{b}}$ Race indicating the avirulence loci for which the isolate is avirulent and has been characterized as proposed in Balesdent et al. 2005. 
shows a nonhost response to L. maculans. Furthermore, as there are different reports about the association between seedling and adult resistance response in B. napus (Li et al. 2003, 2004a; Long et al. 2011; Van de Wouw et al. 2009), further study is required to explore the relationship between $C$. sativa with $L$. maculans.

The genetic basis and mechanisms involved in the resistance of $C$. sativa to L. maculans are not yet well defined or understood. The current studies showed that the HR plays a critical role in this resistance reaction. Previously, the development of HR around the inoculation site has been known as the major characteristic of the resistance to $L$. maculans, both on cotyledons and stems, in Brassicaceae species such as B. napus, A. thaliana, Capsella bursa-pastoris, and Diplotaxis muralis (Chen and Séguin-Swartz 1999; Li et al. 2007b, 2008). This rapid 'suicide strategy' prevents further colonization of L. maculans and therefore the pathogen is confined to an area around the point of inoculation. It is possible that nonspecific activation of defenses in the early hours postinoculation, such as camalexin production, has an essential role in $C$. sativa resistance mechanism against $L$. maculans. A similar mechanism has been reported in $A$. thaliana, in which camalexin production partially contributes to resistance of A. thaliana to L. maculans (Bohman et al. 2004). As the current study confirmed the potential of $C$. sativa as an important source of blackleg resistance, further studies to elucidate the resistance mechanism(s) will almost certainly identify novel R-genes, particularly as the complete genome sequencing data for $C$. sativa is now available (Kagale et al. 2014).

In conclusion, $C$. sativa genotypes exhibited excellent resistance to these two economically devastating pathogens of crucifers. Most genotypes in $C$. sativa showed a level of resistance at or greater than the top-resistance rank with B. napus 'Mystic' and 0P6712 when inoculated with $S$. sclerotiorum and significantly greater resistance than B. napus 'Mystic' when inoculated with L. maculans. C. sativa resistance to these diseases will be a highly valuable source for improvement of crucifers and should also lead to broader adoption of $C$. sativa as a more sustainable oilseed crop in its own right in the future, especially in Australia and other countries where both diseases are devastating to canola and other Brassicaceae crops.

\section{Acknowledgments}

We thank Mr. Rob Creasy for glasshouse support.

\section{Literature Cited}

Agusdinata, D. B., Zhao, F., Ileleji, K., and DeLaurentis, D. 2011. Life cycle assessment of potential biojet fuel production in the United States. Environ. Sci. Technol. 45:9133-9143.

Balesdent, M. H., Barbetti, M. J., Li, H., Sivasithamparam, K., Gout, L., and Rouxel, T. 2005. Analysis of Leptosphaeria maculans race structure in a worldwide collection of isolates. Phytopathology 95:1061-1071.

Barbetti, M. J., Banga, S. K., Fu, T. D., Li, Y. C., Singh, D., Liu, S. Y., Ge, X. T., and Banga, S. S. 2014. Comparative genotype reactions to Sclerotinia sclerotiorum within breeding populations of Brassica napus and B. juncea from India and China. Euphytica 197:47-59.

Barbetti, M. J., Li, C. X., Banga, S. S., Banga, S. K., Singh, D., Singh, P. S., Singh, R., Liu, S. Y., and You, M. P. 2015. New host resistances in Brassica napus and Brassica juncea from Australia, China and India: Key to managing Sclerotinia stem rot (Sclerotinia sclerotiorum) without fungicides. Crop Prot. 78:127-130.

Bohman, S., Staal, J., Thomma, B. P. H. J., Wang, M., and Dixelius, C. 2004. Characterisation of an Arabidopsis-Leptosphaeria maculans pathosystem: Resistance partially requires camalexin biosynthesis and is independent of salicylic acid, ethylene and jasmonic acid signalling. Plant J. 37:9-20.

Bradley, C., Henson, R., Porter, P., LeGare, D., Del Rio, L., and Khot, S. 2006. Response of canola cultivars to Sclerotinia sclerotiorum in controlled and field environments. Plant Dis. 90:215-219.

Buchwaldt, L., Li, R., Hegedus, D., and Rimmer, S. 2005. Pathogenesis of Sclerotinia sclerotiorum in relation to screening for resistance. Paper presented at the Proceedings of the 13th International Sclerotinia Workshop. Monterey, CA.

Campbell, M. C., Rossi, A. F., and Erskine, W. 2013. Camelina (Camelina sativa (L.) Crantz): Agronomic potential in Mediterranean environments and diversity for biofuel and food uses. Crop Pasture Sci. 64:388-398.

Chen, C., and Séguin-Swartz, G. 1999. Reaction of wild crucifers to Leptosphaeria maculans, the causal agent of blackleg of crucifers. Can. J. Plant Pathol. 21:361-367.

DAFWA. 2015. 2015 WA Agribusiness Crop Updates: Research Delivers Opportunities to Cut Canola Production Costs. Department of Agriculture and Food Western Australia, Australia.
Delourme, R., Barbetti, M. J., Snowdon, R., Zhao, J., and Manzanares-Dauleux, M. 2012. Genetics and genomics of resistance. Pages 276-318 in: Genetics, Genomics and Breeding of Oilseed Brassicas. D. Edwards, J. Batley, I. A. P. Parkin, and C. Kole, eds. Science Publishers, CRC Press, USA.

Eynck, C., and Séguin-Swartz, G. 2009. Reaction of Camelina sativa to Sclerotinia sclerotiorum. Can. J. Plant Pathol. 31:483.

Eynck, C., Seguin-Swartz, G., Clarke, W. E., and Parkin, I. A. 2012. Monolignol biosynthesis is associated with resistance to Sclerotinia sclerotiorum in Camelina sativa. Mol. Plant Pathol. 13:887-899.

Fehr, W. 1991. Principles of cultivar development: Theory and technique. Macmillian Publishing Company, New York, USA.

Garg, H., Kohn, L. M., Andrew, M., Li, H., Sivasithamparam, K., and Barbetti, M. J. 2010. Pathogenicity of morphologically different isolates of Sclerotinia sclerotiorum with Brassica napus and B. juncea genotypes. Eur. J. Plant Pathol. 126:305-315.

Garg, H., Sivasithamparam, K., Banga, S. S., and Barbetti, M. J. 2008. Cotyledon assay as a rapid and reliable method of screening for resistance against Sclerotinia sclerotiorum in Brassica napus genotypes. Australas. Plant Pathol. 37:106-111.

Ge, X. T., Li, Y. P., Wan, Z. J., You, M. P., Finnegan, P. M., Banga, S. S., Sandhu, P. S., Garg, H., Salisbury, P. A., and Barbetti, M. J. 2012. Delineation of Sclerotinia sclerotiorum pathotypes using differential resistance responses on Brassica napus and B. juncea genotypes enables identification of resistance to prevailing pathotypes. Field Crops Res. 127:248-258.

Ghamkhar, K., Croser, J., Aryamanesh, N., Campbell, M., Kon'kova, N., and Francis, C. 2010. Camelina (Camelina sativa L. Crantz) as an alternative oilseed: Molecular and ecogeographic analyses. Genome 53:558-567.

GRDC. 2013. Diseases of canola and their management: The back pocket guide. Grain Research \& Development Corporation, Australia.

Gregorich, T. L., Pilgeram, A. L., Peterson, B. L., and Hoch, W. A. 2009. Susceptibility of Camelina sativa to blackleg disease, Leptosphaeria maculans. Paper presented at the Proceedings of 2009 American Society for Horticulture Science Annual Conference. St. Louis, MO.

Gugel, R., and Falk, K. 2006. Agronomic and seed quality evaluation of Camelina sativa in Western Canada. Can. J. Plant Sci. 86:1047-1058.

Iven, T., Hornung, E., Heilmann, M., and Feussner, I. 2016. Synthesis of oleyl oleate wax esters in Arabidopsis thaliana and Camelina sativa seed oil. Plant Biotechnol. J. 14:252-259.

Kagale, S., Koh, C., Nixon, J., Bollina, V., Clarke, W. E., Tuteja, R., Spillane, C., Robinson, S. J., Links, M. G., Clarke, C., Higgins, E. E., Huebert, T., Sharpe, A. G., and Parkin, I. A. P. 2014. The emerging biofuel crop Camelina sativa retains a highly undifferentiated hexaploid genome structure. Nat. Commun. 5:3706.

Khangura, R., and Barbetti, M. J. 2001. Prevalence of blackleg (Leptosphaeria maculans) on canola (Brassica napus) in Western Australia. Aust. J. Exp. Agric. 41:71-80.

Lawrence, R. D., Anderson, J. L., and Clapper, J. A. 2016. Evaluation of camelina meal as a feedstuff for growing dairy heifers. J. Dairy Sci. 99:6215-6228.

Li, C. X., Li, H., Siddique, A. B., Sivasithamparam, K., Salisbury, P., Banga, S. S., Banga, S., Chattopadhyay, C., Kumar, A., Singh, R., Singh, D., Agnihotri, A., Liu, S. Y., Li, Y. C., Tu, J., Fu, T. D., Wang, Y. F., and Barbetti, M. J. 2007a. The importance of the type and time of inoculation and assessment in the determination of resistance in Brassica napus and B. juncea to Sclerotinia sclerotiorum. Aust. J. Agric. Res. 58:1198-1203.

Li, C. X., Li, H., Sivasithamparam, K., Fu, T. D., Li, Y. C., Liu, S. Y., and Barbetti, M. J. 2006. Expression of field resistance under Western Australian conditions to Sclerotinia sclerotiorum in Chinese and Australian Brassica napus and Brassica juncea germplasm and its relation with stem diameter. Aust. J. Agric. Res. 57:1131-1135.

Li, H., Barbetti, M. J., and Sivasithamparam, K. 2003. Responses of Brassica napus cultivars to Leptosphaeria maculans field isolates from Western Australia. Brassica 5:25-34.

Li, H., Barbetti, M. J., and Sivasithamparam, K. 2005. Hazard from reliance on cruciferous hosts as sources of major gene-based resistance for managing blackleg (Leptosphaeria maculans) disease. Field Crops Res. 91:185-198.

Li, H., Damour, L., Sivasithamparam, K., and Barbetti, M. J. 2004a. Increased virulence and physiological specialisation among Western Australian isolates of Leptosphaeria maculans breaking down existing single dominant genebased resistance in six cultivars of Brassica napus. Brassica 6:9-16.

Li, H., Sivasithamparam, K., Barbetti, M. J., Wylie, S., and Kuo, J. 2008. Cytological responses in the hypersensitive reaction in cotyledon and stem tissues of Brassica napus after infection by Leptosphaeria maculans. J. Gen. Plant Pathol. 74:120-124.

Li, H., Stone, V., Dean, N., Sivasithamparam, K., and Barbetti, M. J. 2007b. Breaching by a new strain of Leptosphaeria maculans of anatomical barriers in cotyledons of Brassica napus cultivar Surpass 400 with resistance based on a single dominant gene. J. Gen. Plant Pathol. 73:297-303.

Li, R., Rimmer, R., Buchwaldt, L., Sharpe, A. G., Séguin-Swartz, G., Coutu, C., and Hegedus, D. D. 2004b. Interaction of Sclerotinia sclerotiorum with a resistant Brassica napus cultivar: Expressed sequence tag analysis identifies genes associated with fungal pathogenesis. Fungal Genet. Biol. 41:735-753.

Long, Y., Wang, Z., Sun, Z., Fernando, D. W., McVetty, P. B., and Li, G. 2011 Identification of two blackleg resistance genes and fine mapping of one of these two genes in a Brassica napus canola cultivar 'Surpass 400'. Theor. Appl. Genet. 122:1223-1231.

McKinney, H. 1923. A new system of grading plant diseases. J. Agric. Res. 26: 195-218. 
Neik, T. X., Barbetti, M. J., and Batley, J. 2017. Current status and challenges in identifying disease resistance genes in Brassica napus. Front. Plant Sci. 8:1788.

Nguyen, H. T., Silva, J. E., Podicheti, R., Macrander, J., Yang, W., Nazarenus, T. J., Nam, J. W., Jaworski, J. G., Lu, C., Scheffler, B. E., Mockaitis, K., and Cahoon, E. B. 2013. Camelina seed transcriptome: A tool for meal and oil improvement and translational research. Plant Biotechnol. J. 11: 759-769.

Obour, K. A., Sintim, H. Y., Obeng, E., and Jeliazkov, V. D. 2015. Oilseed camelina (Camelina sativa (L.) Crantz): Production systems, prospects and challenges in the USA Great Plains. Adv. Plants Agric. Res. 2:00043.

Pavlista, A., Isbell, T., Baltensperger, D., and Hergert, G. 2011. Planting date and development of spring-seeded irrigated canola, brown mustard and camelina. Ind. Crops Prod. 33:451-456.

Purnamasari, M., Cawthray, G. R., Barbetti, M. J., Erskine, W., and Croser, J. S. 2015. Camalexin production in Camelina sativa is independent of cotyledon resistance to Sclerotinia sclerotiorum. Plant Dis. 99:1544-1549.

Rana, K., Atri, C., Gupta, M., Akhatar, J., Sandhu, P. S., Kumar, N., Jaswal, R., Barbetti, M. J., and Banga, S. S. 2017. Mapping resistance responses to Sclerotinia infestation in introgression lines of Brassica juncea carrying genomic segments from wild Brassicaceae B. fruticulosa. Sci. Rep. 7:5904

Salisbury, P. 1987. Blackleg resistance in weedy crucifers. Cruciferae Newsl. 12:90.

Séguin-Swartz, G., Eynck, C., Gugel, R. K., Strelkov, S. E., Olivier, C. Y., Li, J. L., Klein-Gebbinck, H., Borhan, H., Caldwell, C. D., and Falk, K. C. 2009. Diseases of Camelina sativa (false flax). Can. J. Plant Pathol. 31:375-386.

Sivasithamparam, K., Barbetti, M. J., and Li, H. 2005. Recurring challenges from a necrotrophic fungal plant pathogen: A case study with Leptosphaeria maculans (causal agent of blackleg disease in Brassicas) in Western Australia. Ann. Bot. 96:363-377.

Sun, J. M., Irzykowski, W., Jedryczka, M., and Han, F. X. 2005. Analysis of the genetic structure of Sclerotinia sclerotiorum (Lib.) de Bary populations from different regions and host plants by random amplified polymorphic DNA markers. J. Integr. Plant Biol. 47:385-395.

Sylvester-Bradley, R. 1984. A code for stages of development in oilseed rape (Brassica napus L.). Asp. Appl. Biol. 6:399-419.

Taylor, A., Coventry, E., Jones, J., and Clarkson, J. 2015. Resistance to a highly aggressive isolate of Sclerotinia sclerotiorum in a Brassica napus diversity set. Plant Pathol. 64:932-940.

Uloth, M., Clode, P. L., You, M. P., and Barbetti, M. J. 2016. Attack modes and defence reactions in pathosystems involving Sclerotinia sclerotiorum, Brassica carinata, B. juncea and B. napus. Ann. Bot. 117:79-95.

Uloth, M., You, M. P., Finnegan, P. M., Banga, S. S., Yi, H., and Barbetti, M. J. 2014. Seedling resistance to Sclerotinia sclerotiorum as expressed across diverse cruciferous species. Plant Dis. 98:184-190.
Uloth, M. B., You, M. P., and Barbetti, M. J. 2015. Host resistance to Sclerotinia stem rot in historic and current Brassica napus and B. juncea varieties: Critical management implications. Crop Pasture Sci. 66:841-849.

Uloth, M. B., You, M. P., Finnegan, P. M., Banga, S. S., Banga, S. K., Sandhu, P. S. Yi, H., Salisbury, P. A., and Barbetti, M. J. 2013. New sources of resistance to Sclerotinia sclerotiorum for crucifer crops. Field Crops Res. 154:40-52.

Van de Wouw, A., Marcroft, S., Barbetti, M. J., Hua, L., Salisbury, P., Gout, L., Rouxel, T., Howlett, B. J., and Balesdent, M. H. 2009. Dual control of avirulence in Leptosphaeria maculans towards a Brassica napus cultivar with 'sylvestris-derived' resistance suggests involvement of two resistance genes. Plant Pathol. 58:305-313.

Waraich, E. A., Ahmed, Z., Ahmad, R., Ashraf, M. Y., Naeem, M. S., and Rengel, Z. 2013. Camelina sativa, a climate proof crop, has high nutritive value and multiple-uses: A review. Aust. J. Crop Sci. 7:1551.

Wei, D., Mei, J., Fu, Y., Disi, J. O., Li, J., and Qian, W. 2014. Quantitative trait loci analyses for resistance to Sclerotinia sclerotiorum and flowering time in Brassica napus. Mol. Breed. 34:1797-1804.

Willbur, J. F., Ding, S., Marks, M. E., Lucas, H., Grau, C. R., Groves, C. L., Kabbage, M., and Smith, D. L. 2017. Comprehensive sclerotinia stem rot screening of soybean germplasm requires multiple isolates of Sclerotinia sclerotiorum. Plant Dis. 101:344-353.

Williams, P. H. 1985. Crucifer Genetics Cooperative (CrGc) Resource Book. University of Wisconsin, Madison.

Wu, J., Cai, G., Tu, J., Li, L., Liu, S., Luo, X., Zhou, L., Fan, C., and Zhou, Y. 2013. Identification of QTLs for resistance to sclerotinia stem rot and BnaC.IGMT5.a as a candidate gene of the major resistant QTL SRC6 in Brassica napus. PLoS One 8:e67740.

Wu, J., Zhao, Q., Liu, S., Shahid, M., Lan, L., Cai, G., Zhang, C., Fan, C., Wang, Y., and Zhou, Y. 2016. Genome-wide association study identifies new loci for resistance to sclerotinia stem rot in Brassica napus. Front. Plant Sci. 7: 1418.

Yin, X., Yi, B., Chen, W., Zhang, W., Tu, J., Fernando, W. G. D., and Fu, T. 2009. Mapping of QTLs detected in a Brassica napus DH population for resistance to Sclerotinia sclerotiorum in multiple environments. Euphytica 173:25-35.

You, M. P., Uloth, M. B., Li, X. X., Banga, S. S., Banga, S. K., and Barbetti, M. J. 2016. Valuable new resistances ensure improved management of sclerotinia stem rot (Sclerotinia sclerotiorum) in horticultural and oilseed Brassica species. J. Phytopathol. 164:291-299.

Zhao, J., Udall, J. A., Quijada, P. A., Grau, C. R., Meng, J., and Osborn, T. C. 2006 Quantitative trait loci for resistance to Sclerotinia sclerotiorum and its association with a homeologous non-reciprocal transposition in Brassica napus L. Theor. Appl. Genet. 112:509-516. 